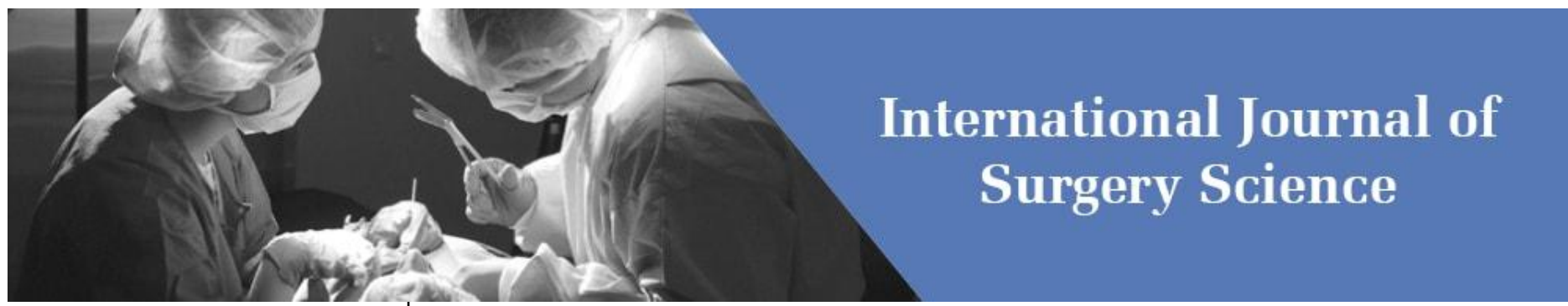

E-ISSN: 2616-3470

P-ISSN: 2616-3462

(C) Surgery Science

www.surgeryscience.com

2019; 3(3): 122-124

Received: 01-05-2019

Accepted: 05-06-2019

Dr. Manjush Kumar Srivastava Associate Professor, Department of Surgery, TS Misra Medical College and Hospital, Lucknow, Uttar Pradesh, India
Correspondence

Dr. Manjush Kumar Srivastava Associate Professor, Department of Surgery, TS Misra Medical College and Hospital, Lucknow, Uttar Pradesh, India

\section{Comparison of laparoscopic and Lichtenstein's hernioplasty in inguinal hernia repair}

\section{Dr. Manjush Kumar Srivastava}

DOI: https://doi.org/10.33545/surgery.2019.v3.i3c.156

\section{Abstract}

Background: Hernia is the abnormal exit of an organ or fatty tissue. The present study was conducted to compare laparoscopic hernia repair and Lichtenstein's hernioplasty in study population.

Materials \& Methods: The present study was conducted on 46 patients of hernia of both genders. Patients were divided into 2 groups of 23 each. Group I patients were treated with laparoscopic repair and group II patients were treated with open Lichtenstein's repair. In both groups, operating time and pain score was compared.

Results: The mean operating time in group I was 90.2 minutes and in group II was 62.4 minutes, pain score was 2.1 in group I and 3.7 in group II, follow up days were 18.2 in group I and 20.4 days in group II. The difference was significant $(P<0.05)$. Associated disorders were diabetes mellitus seen in 2 in group I and 3 in group II, IHD seen 3 in group I and 5 in group II, hypertension seen in 5 in group I and 6 in group II.

Conclusion: Inguinal hernias can be managed with laparoscopic repair and open Lichtenstein's repair. However, laparoscopic repair proves better in terms of pain score.

Keywords: Inguinal hernias, laparoscopic repair, Lichtenstein's

\section{Introduction}

Hernia is the abnormal exit of an organ or fatty tissue, such as the bowel, through the wall of the cavity in which it normally resides. Repair of inguinal hernia is one of the common surgical procedures done worldwide ${ }^{[1]}$ Irrespective of country, race or socioeconomic status hernia constitutes a major health-care drain. The definitive treatment of all hernias, regardless of their origin or type, is surgical repair with approximately 20 million repairs done worldwide annually. The lack of consensus in the literature as to the optimum repair technique or prosthetic mesh to insure a long term durable result is also surprising. The life time risk for men is $27 \%{ }^{[2]}$.

Inguinal hernias present with a lump in the groin that goes away with minimal pressure or when the patient is lying down. Most cause mild to moderate discomfort that increases with activity. A third of patients scheduled for surgery have no pain, and severe pain is uncommon $(1.5 \%$ at rest and $10.2 \%$ on movement). Inguinal hernias are at risk of irreducibility or incarceration, which may result in strangulation and obstruction; however, unlike with femoral hernias, strangulation is rare ${ }^{[3]}$.

Inguinal hernias are often classified as direct or indirect, depending on whether the hernia sac bulges directly through the posterior wall of the inguinal canal (direct hernia) or passes through the internal inguinal ring alongside the spermatic cord, following the coursing of the inguinal canal ${ }^{[4]}$. The present study was conducted to compare laparoscopic hernia repair and Lichtenstein's hernioplasty in study population.

\section{Materials \& Methods}

The present study was conducted in the department of General surgery, TSM Medical College Lucknow. It comprised of 46 patients of hernia of both genders. All were informed regarding the study. Ethical approval was obtained from institute prior to the study.

General information such as name, age, gender etc. was recorded. Patients were divided into 2 groups of 23 each. Group I patients were treated with laparoscopic repair and group II patients were treated with open Lichtenstein's repair. In both groups, operating time and pain score was compared. Results thus obtained were subjected to statistical analysis. P value less than 0.05 was considered significant. 


\section{Results}

Table 1: Distribution of patients

\begin{tabular}{|c|c|c|}
\hline \multicolumn{3}{|c|}{ Total- 46 } \\
\hline Groups & Group I & Group II \\
\hline Procedure & Laparoscopic repair & Open Lichtenstein's repair \\
\hline Number & 23 & 23 \\
\hline
\end{tabular}

Table 1 shows that in group I patients laparoscopic repair and in group II patients open Lichtenstein's repair was done.

Table 2: Comparison of parameters

\begin{tabular}{|c|c|c|c|}
\hline Parameters (Mean) & Group I & Group II & P value \\
\hline Operating time & 90.2 mins & 62.4 mins & 0.01 \\
\hline Pain score & 2.1 & 3.7 & 0.02 \\
\hline Follow up days & 18.2 & 20.4 & 0.62 \\
\hline
\end{tabular}

Table 2 shows that mean operating time in group I was 90.2 minutes and in group II was 62.4 minutes, pain score was 2.1 in group I and 3.7 in group II, follow up days were 18.2 in group I and 20.4 days in group II. The difference was significant $(P<$ $0.05)$.

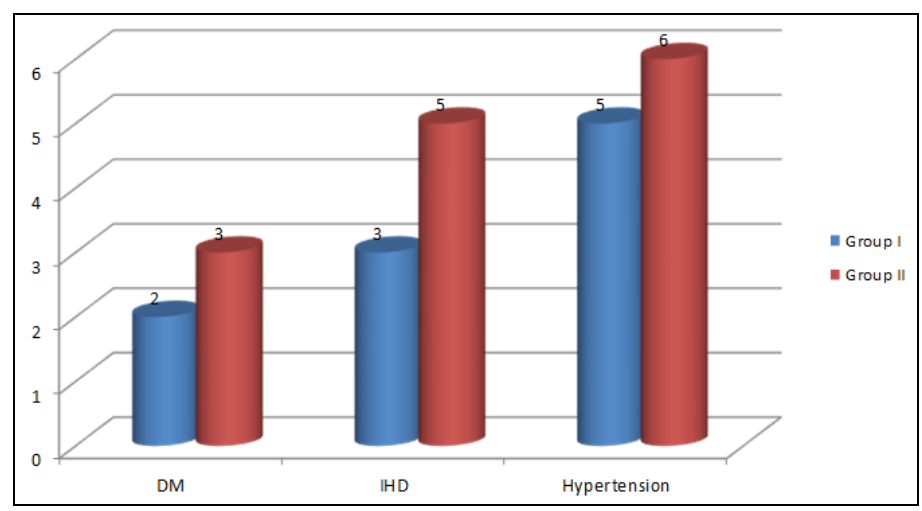

Fig 1: Associated disorders in patients

Figure I shows that associated disorders were diabetes mellitus seen in 2 in group I and 3 in group II, IHD seen 3 in group I and 5 in group II, hypertension seen in 5 in group I and 6 in group II.

\section{Discussion}

Inguinal hernias are one of the most common reasons a primary care patient may need referral for surgical intervention. The history and physical examination are usually sufficient to make the diagnosis. Symptomatic patients often have groin pain, which can sometimes be severe. Inguinal hernias may cause a burning, gurgling, or aching sensation in the groin, and a heavy or dragging sensation may worsen toward the end of the day and after prolonged activity. An abdominal bulge may disappear when the patient is in the prone position ${ }^{[5]}$.

Studies mentioned that laparoscopic hernia repair has got added benefits of lesser pain, reduced discomfort, short hospital stay and early resumption of normal daily activities but still it is not being commonly performed due to need for general anaesthesia and long learning curve ${ }^{[6]}$. Systematic review and meta-analysis of randomized clinical trials have found that, compared with open repair, laparoscopic surgery for hernia is associated with longer operation times but less severe postoperative pain, fewer complications, and a more rapid return to normal activities. Laparoscopic surgery is associated with higher recurrence rates during the learning curve but causes less chronic pain and numbness when assessed by questionnaire up to five years after operation ${ }^{[7]}$. The present study was conducted to compare laparoscopic hernia repair and Lichtenstein's hernioplasty in study population.

In this study, in group I patients laparoscopic repair and in group II patients open Lichtenstein's repair was done. Murthy et al. ${ }^{[8]}$ in their study consisted of 50 patients treated with Hernioplasty (20 cases of laparoscopic hernioplasty and 30 cases of open hernioplasty. Mean operating time in group A was 92.25 minutes while in group B was 43.5 minutes, which is significantly supplementary. Pain score was significantly less in group A with $75 \%$ patients giving score 1-2 (mild pain) and 3 patients with discomforting pain with $P<0.05$.

We found that mean operating time in group I was 90.2 minutes and in group II was 62.4 minutes, pain score was 2.1 in group I and 3.7 in group II, follow up days were 18.2 in group I and 20.4 days in group II. Associated disorders were diabetes mellitus seen in 2 in group I and 3 in group II, IHD seen 3 in group I and 5 in group II, hypertension seen in 5 in group I and 6 in group II. Symptoms of an inguinal hernia may appear gradually over time or develop suddenly, as with incarceration (i.e., the contents of the hernia sac cannot be returned to the abdominal cavity). Inguinal hernias may be asymptomatic and found incidentally on routine physical examination. Symptomatic patients often present with groin pain, which can be severe ${ }^{[9]}$. Stretching or tearing of the tissue at and around the hernia defect can lead to a burning, gurgling, or aching sensation in the groin. This usually causes localized pain directly at the site of the hernia. Pain may worsen with Valsalva maneuvers. Patients may experience a heavy or dragging sensation in the groin, especially toward the end of the day and after prolonged activity ${ }^{[1]}$. Activities that increase intra-abdominal pressure, such as coughing, lifting, or straining, cause more abdominal contents to be pushed through the hernia defect ${ }^{[10]}$.

\section{Conclusion}

Inguinal hernias can be managed with laparoscopic repair and open Lichtenstein's repair. However, laparoscopic repair proves better in terms of pain score.

\section{References}

1. Mahesh GS. Laparoscopic Versus Open Mesh Repair for Inguinal Hernia. Indian Journal of Research. 2015; 11:1046.

2. Bobo Z, Nan W, Qin Q, Tao W, Jianguo L, Xianli H. Metaanalysis of randomized controlled trials comparing Lichtenstein and totally extraperitoneal laparoscopic hernioplasty in treatment of inguinal hernias. J Surg Res. 2014; 192(2):409-20.

3. Pisanu A, Podda M, Saba A, Porceddu G, Uccheddu A. Meta-analysis and review of prospective randomized trials comparing laparoscopic and Lichtenstein techniques in recurrent inguinal hernia repair. Hernia. 2015; 19(3):35566.

4. Smink DS, Paquette IM, Finlayson SR. Utilization of laparoscopic and open inguinal hernia repair: a populationbased analysis. J Laparoendoscopic Adv. Surg Tech. 2009; 19(6):745-8.

5. Bittner R, Schmedt CG, Schwarz J, Kraft K, Leibl BJ. Laparoscopic transperitoneal procedure for routine repair of groin hernia. Br J Surg. 2002; 89(8):1062.

6. McIntosh E. Cost-utility analysis of open versus laparoscopic groin hernia repair: results from a multicentre randomized clinical trial. Br J Surg. 2001; 88(5):653-61.

7. Fitzgibbons RJ, Giobbie-Hurder A, Gibbs JO, Dunlop DD, 
Reda DJ, McCarthy M Jr et al. Watchful waiting vs repair of inguinal hernia in minimally symptomatic men: a randomised clinical trial. JAMA. 2006; 295:285-92.

8. Murthy PK, Ravalia D. Assessment and comparison of laparoscopic hernia repair versus open hernia: a nonrandomized study. Int Surg J. 2018; 5:1021-5.

9. Bisgaard T, Bay-Nielsen M, Christensen IJ, Kehlet H. Risk of recurrence 5 years or more after primary Lichtenstein mesh and sutured inguinal hernia repair. Br J Surg. 2007; 94:1038-40.

10. Hernia EU. Trialists Collaboration. Laparoscopic compared with open methods of groin hernia repair: systematic review of randomized controlled trials. Br J Surg. 2000; 37:860-7. 